\title{
RELACIÓN DE LOS EFECTOS CLIMÁTICOS SOBRE LAS ESTRATEGIAS DE PRODUCCIÓN DE TOMATE (LYCOPERSICUM ESCULENTUM MILL) EN EL VALLE DE AZAPA, PROVINCIA DE ARICA, ENTRE LOS AÑOS 1995-20051
}

\author{
WEATHER EFFECTS OVER TOMATO PRODUCTION STRATEGIES \\ (LYCOPERSICUM ESCULENTUM MILL) IN AZAPA VALLEY, PROVINCE \\ OF ARICA, BETWEEN THE YEARS 1995 AND 2005
}

\author{
Arlette Saavedra C. ${ }^{2}$; Luis Tapia I. ${ }^{3}$
}

\begin{abstract}
RESUMEN
Este trabajo analiza los antecedentes que intervienen en la definición de la estrategia de producción de tomate en el valle de Azapa, la cual se enmarca como una estrategia de producción de contra estación, que puede generar resultados muy favorables cuando las condiciones climáticas en la zona centro sur desarrollan años fríos o muy fríos, que se expresan en más de 1.000 horas de frío para la temporada. En esta situación los costos de producción, en el caso de los productores de la V Región, se incrementan significativamente por la necesidad de calefaccionar sus naves productivas; dichos costos compiten con los costos de transporte de los tomates a enviados desde el norte del país.

La toma de decisiones por parte de los productores del valle de Azapa y sus competidores directos, principalmente los productores de la localidad de Quillota, son hechos que se presentan en forma simultánea; de ahí la importancia de conocer los registros de las horas de frío en la V Región, que se expresan entre los meses de marzo a junio, a fin de contar con antecedentes básicos a ser considerados por los productores del norte del país para tener éxito en la participación de los mercados mayoristas de la zona central del país.
\end{abstract}

Palabras clave: Producción de tomate del valle de Azapa, condiciones de contra estación, competidores V Región Chile.

\begin{abstract}
This paper analyzes the basic antecedents to establish the strategies of tomato production in the Azapa valley, under off-season conditions. These strategies are favorable to the northern farmers when the winter time in central area of Chile is too cold with over 1000 hours of cold weather conditions. Under this situation the costs of production for the growth of tomatoes in the 5th Region increase, because they need to raise the temperature in the green house. These costs compete with the transportation costs of tomatoes sent from the Azapa valley.

The decisions made by the Azapa farmers and mainly by their competitors, the ones of the 5th Region, are generated at the same time. For this reason it is important to know the register of cold hours in the 5th Region between March and June, to establish successful strategies of tomato production with high participation in the important market of the central area of Chile.
\end{abstract}

Key words: Tomatoes production in the Azapa valley, off season production, competitive farmer in V Regions Chile.

\footnotetext{
1 Trabajo desarrollado en el marco de la Memoria de Título "Evaluación de las estrategias de producción de tomate (Lycopersicum esculentum Mill) fuera de estación para la agricultura de la provincia de Arica, Chile, entre los años 1995-2005".

2 Facultad de Ciencias Agronómicas, Universidad de Tarapacá.

3 Facultad de Ciencias Agronómicas, Universidad de Tarapacá, E-mail: 1tapia@uta.cl
}

Fecha de Recepción: 5 Junio 2009

Fecha de Aceptación: 20 Julio 2009 


\section{INTRODUCCIÓN}

El tomate es el principal producto hortícola del valle de Azapa, con una producción continua durante todo el año debido a las favorables condiciones climáticas que se presentan y que han permitido históricamente abastecer al mercado nacional durante el periodo invernal.

La temperatura del periodo invernal se transforma entonces en una ventaja comparativa para los productores de tomates de la provincia de Arica, debido a la estacionalidad que presenta el mercado de tomate fresco en el país.

Es así como Huber (1978) a partir de los antecedentes sobre potencial fotosintético fundamenta que las estrategias de producción de hortalizas fuera de estación que se presentan en la zona norte de Chile y se expresan en la diferencia del potencial fotosintético, se traduce en $2,0 \mathrm{~kg}$ de materia $\mathrm{seca} / \mathrm{m}^{2} / \mathrm{año}$ en la zona norte, disminuyendo a $1,5 \mathrm{~kg}$ de materia seca $/ \mathrm{m}^{2} /$ año en la zona central y con un escaso $1,0 \mathrm{~kg}$ de materia seca/m2/año en la zona sur.

La provincia de Arica con un clima subtropical produce tomate al aire libre desde mayo a noviembre abasteciendo durante este periodo con aproximadamente un $90 \%$ de tomate en el mercado nacional de contraestación. Es el mercado de la zona central el que presenta mayores atractivos para los productores ariqueños, donde las hortalizas fuera de estación obtienen altos precios y donde, además, se concentra el grueso de la población del país (ODEPA, 2004).

Para los productores de tomate de contraestación de la provincia de Arica, la competencia directa proviene de las regiones III, IV y V. Los primeros han mostrado una interesante expansión en los últimos años, mientras que los productores de la V Región han aumentado la superficie de producción bajo invernadero en un $50 \%$ entre los años 1995-2005 (ODEPA, 2006).

La Región de Valparaíso representa una de las zonas con mayor concentración de producción de tomate bajo invernadero del país, ubicada en su mayoría en las localidades de Limache, Olmué y Quillota. La tecnología empleada les permite la obtención de tomate a finales de invierno y principio de primavera, participando en el abastecimiento de los principales mercados mayoristas de la Región Metropolitana durante los meses de septiembre a diciembre. Op cit.
La producción de tomate fuera de estación significa aprovechar las ventajas comparativas que posee la provincia de Arica, que es el clima. No obstante esta actividad, el mercado interno enfrenta una seria competencia con la producción de tomate bajo invernadero de la zona central, donde el uso de sistemas forzados que incluye un alto nivel tecnológico que supone la calefacción para obtener temperaturas superiores a los $10^{\circ} \mathrm{C}$ ha permitido mejorar su producción fuera de época disminuyendo las ventajas competitivas de la zona norte.

Las estrategias de producción desarrolladas por los productores de tomate del valle de Azapa entre los años 1995-2005 apuntan a innovaciones tecnológicas, lo que hace referencia a la tecnificación del riego, incorporación de nuevas variedades híbridas con resistencia a ataques de nematodos y con mayor capacidad de almacenaje, capacitación de sus trabajadores y mejora en la gestión comercial, Rojas (1997). Esto implica que ya no basta la simple ventaja climática, sino que además se debe mejorar la técnica de producción a través de sus estrategias para incrementar la oferta provincial en periodos de altos precios y aumentar así la rentabilidad.

La contrastación empírica indica que las variaciones de los volúmenes de producción de tomate en el valle de Azapa tienen directa relación con las variaciones de las horas de frío (HF) registradas en la localidad de Quillota durante los años 1995-2005.

\section{MATERIALES Y MÉTODOS}

El estudio realizado es de tipo descriptivo, cuyo propósito es analizar la situación de las estrategias de los productores de tomate en el valle de Azapa, provincia de Arica, y caracterizar los efectos climáticos como una variable que incide en la toma de decisiones llevada a cabo por los productores locales. Pero a su vez es una investigación de carácter explicativo donde se evaluará el comportamiento de un conjunto de variables (Volúmenes enviados, precios y horas de frío).

El diseño del estudio es de tipo documental, con medición de variables a partir de datos secundarios, Hernández et al. (2003). El propósito de la investigación es de tipo aplicada a la solución de problemas prácticos que enfrenta la competitividad del sector hortícola, específicamente el rubro tomate, en el valle de Azapa, provincia de Arica, en condiciones de contraestación. 
El estudio se realizó a partir de series de datos de volumen de tomates arribados a los mercados mayoristas de Santiago provenientes de la provincia de Arica e información de horas fríos media anuales registradas en la localidad de Quillota, V Región. Esta información se recopilo a partir de base datos de la Oficina de Estudios y Política Agraria (ODEPA, 1995-2005) y el Centro Regional de Investigación Agroclimatológica de la V Región (CRIA, 19952005). Los resultados fueron analizados mediante estadísticas paramétricas utilizando el programa SPSS, versión 10.0 (Analytical software).

\section{RESULTADOS Y DISCUSIÓN}

El mercado nacional de tomate se presenta con una oferta más regular en el año. Tal situación se debe en parte a los envíos de tomate desde la zona norte específicamente de la provincia de Arica y al aumento de la superficie cultivada en invernaderos en la V Región. Las tecnologías aplicadas hoy hacen posible que se logren mayores rendimientos del cultivo y la posibilidad de obtener producciones durante el periodo invernal.

La distribución mensual de la producción de tomates fuera de temporada para las distintas localidades se muestra en la Figura 1.

En la Figura 2 se presenta la evolución de los volúmenes de tomates enviados a los mercados mayoristas de Santiago durante el decenio 1995 -2005. Este presenta una tendencia lineal con pendiente positiva, lo que está relacionado con las estrategias seguidas ( tecnología y comercialización) por los productores de tomate del valle de Azapa a fin de mantener el liderazgo en el abastecimiento del producto en el período invernal en la Región Metropolitana. Es así como se puede observar el aumento de los envíos en los últimos años, 20032004, registrándose volúmenes sobre las 1.200 t; el año 2001 también se observa un volumen considerable de aproximadamente $1.000 \mathrm{t}$. En cuanto a los menores envíos estos corresponden a los años 1996-1997, donde sólo se registró un volumen de aproximadamente $200 \mathrm{t}$; por otra parte, la mayor caída en los envíos se produjo entre los años 20042005 con una diferencia de $700 \mathrm{t}$.

Esta tendencia además indica la conducta de los consumidores de reducir la estacionalidad en el consumo durante el período invernal conectado al sostenido aumento de los ingresos destinados al consumo de este producto.

El valor estimado del coeficiente de correlación de Pearson es de un 0,67, lo que nos indica una alta correlación entre las variables volumen y el tiempo en que se realizan estos envíos a los mercados mayoristas de Santiago. Este valor de la correlación es significativo a un nivel de confianza del 5\% (Little y Hills, 1976).

En la Figura 3 se presenta la evolución de los registros históricos de horas de frío media anuales de la localidad de Quillota, en la V Región de Chile, entre los años 1995-2005. La tendencia lineal muestra una pendiente positiva, que se refleja con buenas condiciones climáticas durante los años 1996, 1997 . 1999 y 2000 durante el decenio; la variación de temperaturas en Quillota se relaciona con los cambios climáticos que se presentan a nivel mundial y que se expresan en esa zona del país. Como se puede observar, las condiciones climáticas desfavorables

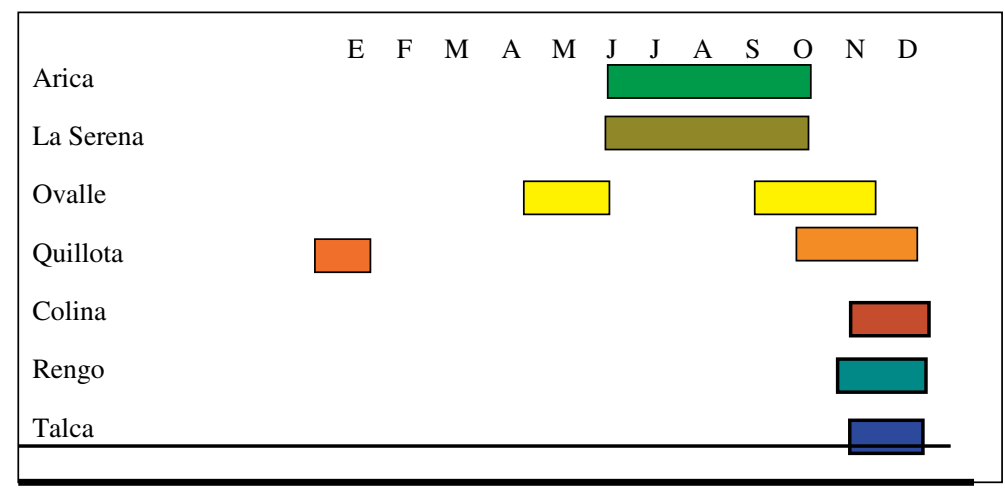

Figura 1. Localidades y épocas de producción de tomates. Fuente: Elaboración propia a partir de base de datos de ODEPA. 


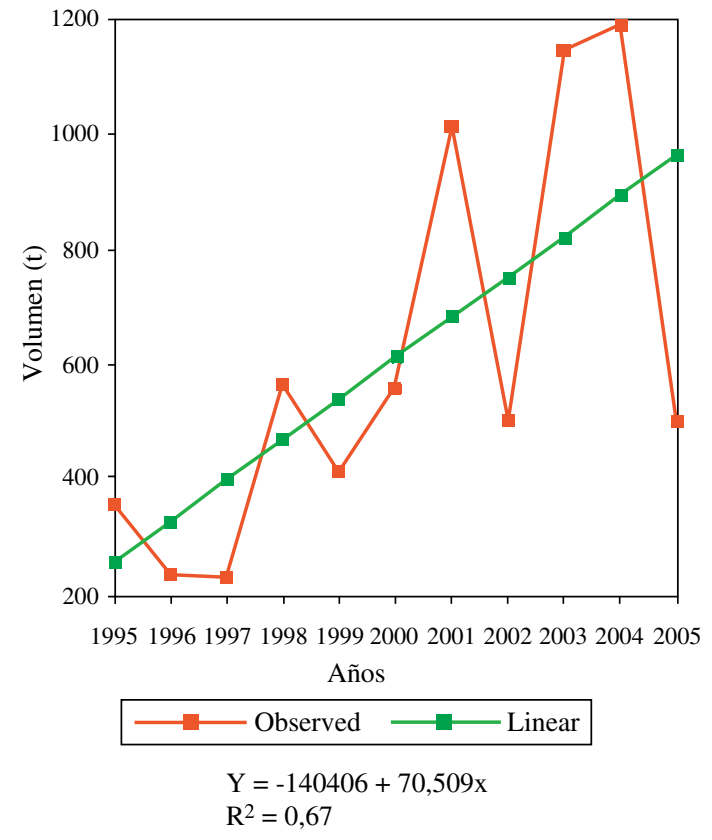

Figura 2. Evolución de los volúmenes de tomate fresco enviados a los mercados mayoristas de Santiago período 1995-2005. (expresado en toneladas).

Fuente: Elaboración propia a partir de datos de la Oficina de Estudios y Políticas Agrarias (ODEPA) 1995-2005.

en la localidad se presentan durante los años 1995, 1998,2001 y 2005 y con acumulaciones mayores 1.000 horas de frío.

El coeficiente de correlación lineal de Pearson de 0,28 indica que no existe correlación entre la variable Horas de Frío media anuales de la localidad de Quillota y el volumen de tomate arribado a los principales mercados mayoristas de Santiago. Este valor de la correlación no es significativo a ningún nivel de confianza, Little y Hills, op cit. (1976).

Como se observa en la Figura 4, el tamaño de la brecha entre los volúmenes de tomate enviados desde la provincia de Arica y las horas de frío (HF) media anuales de la localidad de Quillota se estrecha durante los años 1999 y 2000, donde la presencia de los tomates provenientes de Quillota aumentó la oferta de contraestación en los mercados mayoristas de Santiago. Entre las causas de este aumento se encuentran las altas temperaturas registradas en el periodo invernal, lo cual permitió la obtención temprana de la producción de tomate en dicha localidad, generando una reducida brecha para la colocación de tomate proveniente de la provincia de Arica.

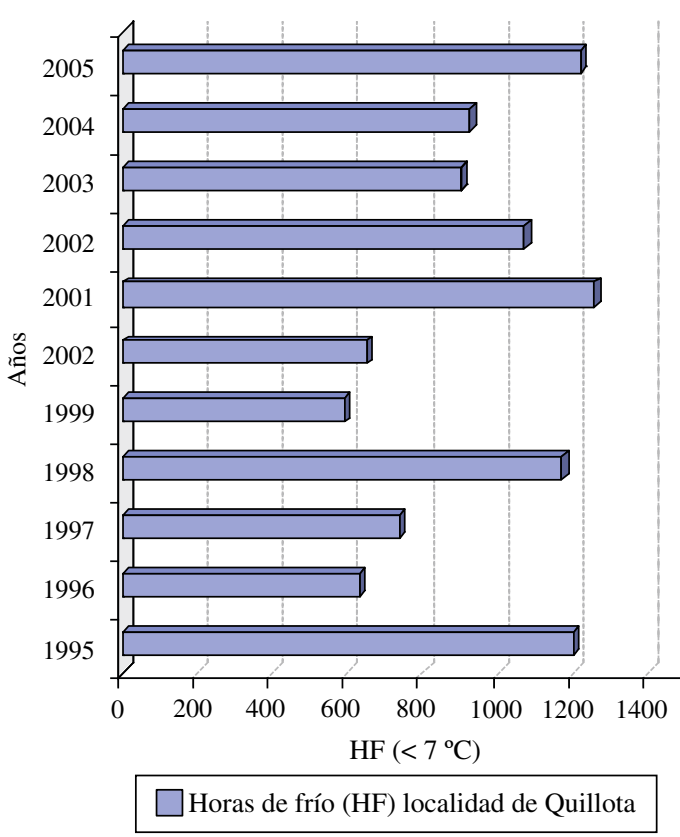

Figura 3. Evolución de los registro históricos de horas de frío media anuales de la localidad de Quillota, V Región, período 1995-2005 (expresado en grados C).

Fuente: Elaboración propia a partir de base de datos del Centro Regional de Investigación Agroclimatológica (CRIA), V Región, 1995-2005.

Las bajas temperaturas y por ende las malas condiciones climáticas registradas en Quillota durante los años 1995, 1998, 2002 y 2005 aumentaron considerablemente la brecha entre los volúmenes enviados y las horas de frío observadas en la localidad de la V Región, situación que aumenta la participación de mercado para los tomates producidos en Quillota, como se puede apreciar en la Figura 4. Si bien los volúmenes arribados a los mercados mayoristas de Santiago provenientes de la provincia de Arica son bajos en comparación a los restantes años, la baja en los volúmenes de tomates no tendría relación con la competencia de los tomates procedentes de la V Región, sino a otros factores como la firma de acuerdos comerciales con Perú y que permitió el ingreso de tomate fresco desde el año 2001, con el levantamiento de las barreras fitosanitarias existentes para la I Región por presencia de la mosca de la fruta (Ceratitis capitata).

\section{CONCLUSIONES}

La producción de tomate fuera de estación en la provincia de Arica tuvo como principal destino 
Horas de frío - Volumen

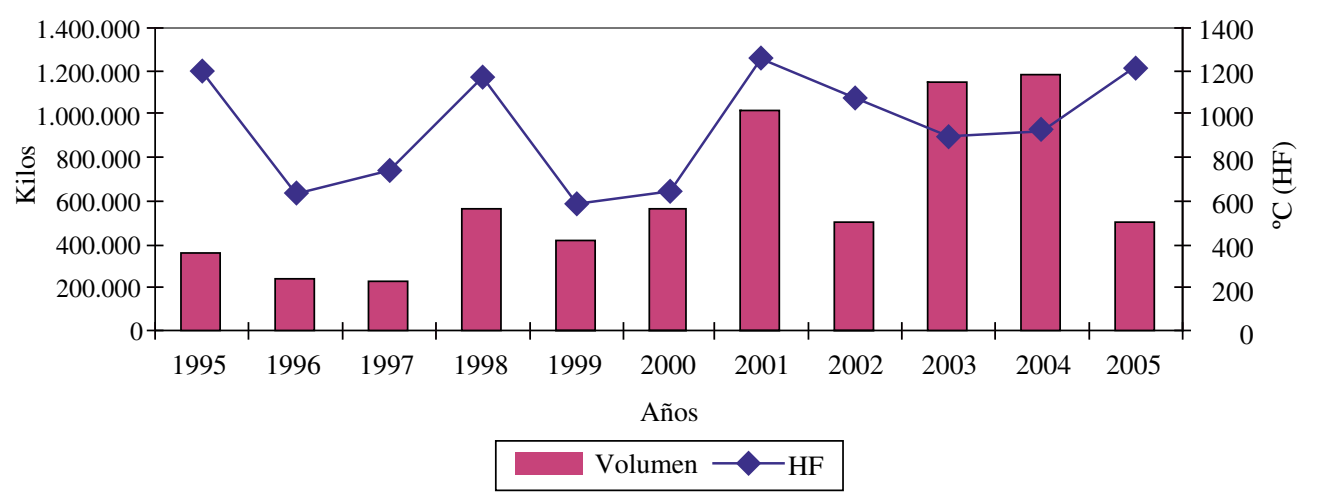

Figura 4. Horas de frío media anuales localidad de Quillota y volúmenes de tomates frescos arribados a los mercados mayoristas de Santiago provenientes Arica. Categoría primera, unidad de comercialización caja de 18 kg, entre los años 1995-2005. Fuente: Elaboración propia a partir de base de datos de ODEPA y CRIA, 1995-2005.

los mercados mayoristas de Santiago (Central Lo Valledor, Vega poniente y Mapocho Venta Directa) entre los años 1995-2005. La incorporación paulatina de estrategias de producción y comercialización durante esta última década en los procesos productivos permitió por una parte en materia de riego tecnificar este sistema, utilizando riego por goteo y la distribución eficiente del recurso hídrico. Por otra parte, la incorporación de nuevas variedades híbridas que favoreció el aumento del rendimiento por sobre las $120 \mathrm{t} / \mathrm{ha}^{-1}$ al aire libre, además de contar con un producto de mayor vida útil en poscosecha y de esta forma llegar a los centros de abastecimiento sin perder las características organolépticas del producto. Las continuas innovaciones tecnológicas en materia de producción han generado condiciones que a diferencia de sus competidores de las regiones III, IV y V, le han permitido controlar el $95 \%$ del abastecimiento de tomate en el mercado nacional durante los meses de agosto y septiembre.

Las bajas de temperatura que afectan a las localidades productoras de tomate de la $\mathrm{V}$ Región aumentan considerablemente los costos de su producción bajo los sistemas invernadero; las continuas heladas obligan a los productores a utilizar calefacción como método de producción para evitar que las temperaturas desciendan por debajo del punto de congelación. Bajo estas condiciones la distancia de la brecha entre los envíos de volumen de tomate desde la provincia de Arica y las horas fríos registradas en la localidad de Quillota, se estrecha cuando las condiciones climáticas invernales son normales, vale decir, en ausencia de heladas, de esta forma la participación de mercado o la presencia de volúmenes durante los meses de septiembre, octubre y noviembre repercute en los envíos de tomate desde el valle de Azapa, siendo menor su abastecimiento para ser transado en los mercados mayoristas de Santiago. Por el contrario, las temperaturas por debajo de cero grados durante los meses de inviernos obligan a los productores de la localidad de Quillota a aumentar los costos de producción en calefacción, permitiendo llegar en los meses de octubre y noviembre, por ende, la distancia de la brecha aumenta, lo que permite a productores del valle de Azapa liderar el abastecimiento durante los meses de menor oferta de tomate y alcanzar los mayores precios.

Los procesos climáticos que se registran en la V Región, específicamente la localidad de Quillota y la toma de decisión de parte de los agricultores del valle de Azapa de producir tomate fuera de estación, son hechos que se presentan en forma simultánea. Una de las estrategias a seguir de parte de los productores es llevar un registro de las horas de frío que se presentan entre los meses de marzo a junio en la V Región en el año que se desea producir y de esta manera en los años con horas de frío superiores a 1.000 se consideran favorables para la producción de tomate en condiciones de contraestación en el valle de Azapa; por el contrario, años con horas de frío bajo las 1.000 serían condiciones desfavorables para producir y aumentar las superficies de tomate fuera de estación, lo que causaría que la rentabilidad de los productores de tomate del valle de Azapa disminuya por el aumento de oferta proveniente de la V Región a los mercados mayoristas de Santiago durante la época de contraestación. 


\section{LITERATURA CITADA}

HERNÁNDEZ R., FERNÁNDEZ C., BAPTISTA P. 2003. Metodología de la Investigación. McGraw-Hill. México. 64-575 p.

HUBER D. 1978. La productividad potencial en la vegetación natural de Chile. Revista Agrosur No 6 (2): 83-89 p.

LITTLE, T. \&. HILLS, F. 1976. Correlación y regresión para más de dos variables En: Métodos estadísticos para la investigación para la agricultura. Ed. Trillas México. 206-218 p.

OFICINA DE ESTUDIOS Y POLÍTICAS AGRARIAS

(ODEPA). Base de datos de volumen de tomate mercados mayoristas de Santiago. Chile. Disponible en: http://www. odepa.gob.cl
OFICINA DE ESTUDIOS Y POLÍTICAS AGRARIAS (ODEPA) 2004. Mercado de hortalizas en Arica. Consulta: 15 de mayo 2006. En: http://www.odepa.gob.cl. (02 de julio 2004).

CENTRO REGIONAL DE INVESTIGACIÓN AGROCLIMATOLÓGICA (CRIA). Base de datos de horas fríos anuales, Quillota. Chile. Disponible www. meteochile.cl/agro_centros.html

ROJAS, L. 1997. Producción de primores de alta calidad, una buena alternativa para el norte de Chile En: Anuario del campo. INIA. Ed: Publicaciones Lo Castillo. 110$116 \mathrm{p}$. 